\title{
Mother-Grandmother Co-Parenting in Multigenerational Urban Family in Indonesia
}

\author{
Dewi Ilma Antawati ${ }^{*}$
}

\author{
${ }^{1}$ Faculty of Psychology, University of Muhammadiyah Surabaya, Surabaya, Indonesia \\ *Corresponding author.Email: dewi_raditya@fpsi.um-surabaya.ac.id
}

\begin{abstract}
Multi-generational families are common in Indonesia, but research in this context is still very rare. This study is conducted to understand how grandparents and parents in the context of multi-generational families jointly carry out childcare using a qualitative case study approach. In-depth interviews were conducted with grandparents and parents from the lower-middle class who lived together (co-residence) and had children under 7 years old in urban area of Surabaya. The results of the interviews were then analyzed using interactive analysis techniques from Miles and Huberman. The results of the interviews show that family care is the main responsibility and carried out jointly by the mother and grandmother, while the men in the family act as the head of the family, decision-makers, and providers of the main financial needs. Some families can do co-parenting well, but some mothers and grandmothers face conflict in doing co-parenting. This research raises questions for further research in the context of multigenerational families.
\end{abstract}

Keywords: co-parenting, multigenerational family, parenting

\section{INTRODUCTION}

Human development is the result of the interaction between humans and their environment. The first environment in which individual development occurs is the family. In research on families, human behavior scientists [1] [2] [3] [4] have considered the influence of diversity in family structure and patterns of family interaction in individual development. In Indonesia, it is common to find grandparents who live with nuclear families. Families consisting of several generations (children, parents, grandparents) are also referred to as multigenerational families [5] [6].

Mindel [5], defines multigenerational families as families consisting of three or more generations living together and two generations of families where parents of one or both partners are present in the family or one generation is absent (for example when grandparents and grandchildren live together). Multigenerational families also include families where siblings of grandparents live with nuclear families, for example, siblings or cousins. However, Bengtson [6] limits multigenerational families to families consisting of nuclear families and grandparents who live together.

No data explains in detail about the number of multigenerational families in Indonesia, but the data can be seen from the number of elderly people who live together in families of three generations in one household (Table-1).

In Table-1 we can see the percentage of the number of elderlies who live together in three generations of families from 2015 to 2018. The percentage of the total number had decreased in 2016, but in the following years, the number has increased. When compared by type of region, there is only a slight difference in the number of elderly people in villages compared to the elderly in cities who live with families of three generations. The small difference in the number shows the value of a collectivistic family in Indonesia. Indonesian culture emphasizes that everyone is responsible for his family, especially his parents. Children are required to respect and serve their parents; one form of respect is to take care of them when they are old. Entering the elderly into a nursing home is considered a negative action, which is not thanking the parents or disrespecting the parents. Thus, the elderly is more supported if they live alone or live with family. Based on sex, more elderly women live with families of three generations than older men. This shows a woman's life expectancy is higher than men [10].

Table I Percentage of Elderly According to Three Generations Living Arrangement

\begin{tabular}{|lllll|}
\hline \multirow{2}{*}{ Demography } & \multicolumn{4}{l|}{ Percentage per Year } \\
\cline { 2 - 5 } & 2015 & 2016 & 2017 & 2018 \\
\hline Total & 35,62 & 33,92 & 35,73 & 43,18 \\
Region Type & & & & \\
$\quad$ Urban & 17,07 & 17,20 & 18,03 & 21,92 \\
$\quad$ Rural & 18,55 & 16,72 & 17,70 & 21,26 \\
Sex & & & & \\
$\quad$ Male & 14,85 & 14,17 & 15,13 & 17,93 \\
$\quad$ Female & 20,77 & 19,75 & 20,60 & 25,25 \\
\hline
\end{tabular}

The condition of a multigenerational family is an ideal condition for the elderly but can also create complex problems. On the one hand, the elderly gets economic and social support from their families and vice versa the elderly can also provide social support related to the care of children (the grandchild of the elderly) [11]. In the parenting process, the presence of grandparents in multigenerational families can influence grandchildren directly when they act as caregivers, playmates, and successors of family history who pass on information that can strengthen a sense of sustainability between generations. They have a direct effect when acting as a mentor to their grandchildren and 
they help negotiations between parents and children. They affect grandchildren indirectly when they provide psychological and material support for parents, from which support parents have more resources to care for [12]. Support from grandparents is related to more adaptive family conditions and is a protective factor for families with interdependent cultures such as African-American, Latin, and Asian families. Grandparents' support can also make the quality of parental cooperation between fathers and mothers better so that families become more adaptive [13].

On the other hand, the co-existence of the elderly with extended families in addition to generating solidarity can also lead to conflicts, especially conflicts between parents and children. Bengston et al. [14], state that the paradox between conflict and solidarity is characteristic of most parent-child relationships. Lüscher \& Pillemer called it intergenerational ambivalence [15]. This ambivalence will be seen when the two generations carry out a joint task, for example, childcare. When two generations take care of a child together, the mother simultaneously carries out the roles of child and mother, while the grandmother takes on the role of parent and grandmother, so the boundary between the subsystems becomes ambiguous or ambivalent. An imbalance of power in the relationship between mother and grandmother can create conflict between mothers and grandmothers which in turn poses a direct and indirect risk to the social development of children when they are cared for together [4].

Parenting cooperation by two or more caregivers is called co-parenting. In general, co-parenting is defined as a collaboration between two caregiver figures in carrying out childcare responsibilities [16]. Some researchers mention co-parenting with different terms, such as shared parenting [17], parenting partnership [18], and parenting alliance [19]. Even though co-parenting is always associated with the life domain of married couples, it does not mean that coparenting is only done by them. The broader definition shows that co-parenting also occurs in other family structures. Van Egeren \& Hawkins [20] explain that coparenting relationships arise when two individuals have mutual agreement or based on social norms have a shared responsibility for the welfare of children. Thus, coparenting can occur in couples who are married, not married, or divorced, regardless of the sexual orientation of the couple, and do not see the biological relationship of children with caregivers.

Several studies and literature studies show the role of coparenting between mothers and grandmothers in shaping maternal parenting behavior and influencing child development. Research Baker et al of 40 pairs of grandmothers and mothers who are in prison [21], by observing when they are discussing children. The results of this study indicate that good co-parenting relationships are associated with externalizing behavior problems in children. Barnett et al, examined 117 African American families from lower socioeconomic classes who have preschool or early elementary school children [22]. The results show that there is a positive relationship between coparenting to positive parenting practices by mothers towards the oldest child, and the social competencies of the oldest and younger children. Li \& Liu, examine the relationship between co-parenting parents/grandparents with children's social competence and maternal parenting self-efficacy [23]. The results show that maternal parenting self-efficacy partially mediates the relationship between coparenting mothers and grandmothers with children's social competence. The harmonious co-parenting relationship between mother and grandmother directly influences significantly on good social competence in children, because the harmonious relationship is an example for children to interact with others. The co-parenting relationship between mother and grandmother can also affect a child's social competency indirectly through maternal parenting self-efficacy, where grandmothers provide support to share experiences, show how to deal with parenting problems, and provide adequate feedback to mothers so that mothers have good parenting self-efficacy. Mothers who have good parenting self-efficacy are more likely to succeed in building warm and sensitive relationships with children, able to understand the child's needs and respond accordingly. Spencer et al. examined the relationship between multigenerational correspondence, intrafamilial conflicts related to childcare, and parental stress in 190 adolescent mothers at 6, 12 and 24 months [24]. As a result, conflicts that persist until a 24-month-old child is associated with high levels of parental stress on mothers. Barnett et al, examined the relationship between symptoms of caregiver depression, verbal conflict between mother-grandmother, caregiving behavior, and prosocial behavior in 85 multigenerational families from lower economic classes who have 36-month-old children [22]. The results show that conflicts in the relationship between mother and grandmother pose an indirect risk to the child's behavior through negative parenting behavior.

Unfortunately, research related to co-parenting is still very limited in Indonesia. Yet according to Bornstein culture forms the construct of parenting by maintaining and transmitting beliefs and behaviors related to parenting [25]. The consequences for different cultures of parenting behavior will also be different because one of the goals of parenting is to prepare children to be part of the community. For this reason, the authors conducted a study to obtain a picture of co-parenting, especially between mothers and grandmothers in multigenerational families as a common form of family in Indonesia.

\section{METHOD}

This research was conducted using a qualitative approach to the type of instrumental case study to get an in-depth explanation of the case under study. The theoretical perspective used to explain the subject's co-parenting behavior is the theoretical perspective of co-parenting [16] [26]. The use of theoretical perspectives from the two researchers is carried out to see whether the theories are appropriate to be used to explain the co-parenting behavior in Indonesia. First, in-depth interviews were conducted with mothers who have children aged 7 years and under, with a lower-middle economic background, but when the analysis was conducted after interviewing the mothers, it was found that the mother carries out child care activities with grandmother. Therefore, the researcher interviewed the grandmother as the mother's co-parenting partner. The 
selection of subjects from lower-middle socioeconomic classes is done because children from families with an unfavorable economic background are said to be at risk of experiencing maladaptive social-emotional development, including high behavioral problems and low social skills [27] [28]. Subjects were obtained through an intermediary from an early childhood education centre teacher in Surabaya, and interviews were conducted at each subject's home, which is in the urban area in Surabaya.

The interview was conducted in the morning to coincide with a national holiday. Through interviews with mothers, it was found that all mothers lived with their biological mother, none of them live with their mother in law. It was also found that both fathers and mothers of all families work outside the home with various working hours, some work on a shift system. Interview results were analyzed using the Interactive Model approach, which consisted of three stages, namely data reduction, data presentation, and concluding [29]. In the data reduction stage, the author sharpens, classifies, directs, discards unnecessary, and organizes data in such a way that the conclusions can be drawn and verified. At the presentation stage, the writer arranges information into matrix form to combine information that has been classified based on certain themes so that the researcher can decide to draw correct conclusions or continue taking data. The stage of concluding is not only done at the end of the data analysis process, but since researchers note, discover patterns, and continue to develop along with the discovery of new data and new understanding.

\section{RESULT}

\subsection{Background and reasons for choosing to live together}

All subjects lived with their daughter, son-in-law, and grandchildren. Children and son-in-law of all three subjects work outside the home. One mother work full time, while three mothers working in the shift system. With the condition of the mothers and fathers working, care is mostly carried out by the grandmother, but the responsibility of care is shared between the daughter and mother. In subject 1 (grandmother1) care was more carried out by subjects than children and their son-in-law, whereas in grandparents' subjects number 2 and 3, care was carried out alternately, but more portions were done by grandmothers. Son-in-law or grandfather do not share the responsibility of caring. A son-in-law is only involved when there is time off and is limited to playing with children, not doing daily maintenance.

Well.. His father decide. I don't know about the plan, but he said he has plan to send him (her grandson) to islamic boarding school. (NY/030419. $68-71)$

All three subjects decided to live with their children and grandchildren because of economic reasons and the desire to care for and be closer to grandchildren. Subjects 2 and 3 live in the subject's parents' inheritance house, while subject 1 lives in the subject's parents-in-law's inheritance house.
Even if the family is capable of employing household assistants or child care givers, there is mistrust they have, as expressed by NY:

Yeah, you know $i$ often watch news in television that some kids was treated badly by the nanny, well never mind, $i$ take care of them myself even though I tired but $i$ 'm happy, taking care of grandchild is tiring but fun, heheh (NY/030419. 53-55)

Mothers entrust their child care for grandmothers because they consider grandmothers more experienced so that they are more able to do care and know more about care than the mothers. Mothers who are married and have children at a young age also feel unsure of their ability to carry out care, so they are more confident when the grandmothers caring for their children.

\subsection{Child characteristics and desired child quality}

All six subjects prioritize adherence and the child's ability to control their behavior. This can be seen from the subject's expectation that the child is obedient to adult directives. Problems raised by subjects related to children's behavior also indicate this, namely, the child is fussy when asking for something. There are two definitions of fuss intended. The first child is considered to talk a lot, often argue, and have many requests that sometimes cannot be fulfilled by caregivers. When the request is not obeyed the child will cry and get angry. Secondly, children are considered difficult to be directed to follow certain routines or activities, such as wearing shoes when participating in activities outside the home, or always playing cellphones when they come home from school and ignoring other activities.

One pair of mothers and grandmothers also expressed pride in children who were able to be independent and able to control their behavior, such as putting things in their place when told to or being able to refrain by not showing explosive emotions and negotiating when their requests were not met by adults. Subjects 1 and 3 have the same assessment of each grandchild, where their grandchildren are considered spoiled and rather difficult to follow the routine and tend to behave as they wish. In contrast to the grandchildren of Subject 2, who is more independent, has controlled behavior, can hold back when his desires are not fulfilled and can negotiate when his desires are not fulfilled.

It's easier for taking care of her sister ... compare to children nowadays... children now are super active, absolutely super. His sister always obey what I say. But the children no, oh my.. So it needs ... what do you need. you need patience.. (MA/030419. 72-76)

Well.. every parent must be hoping that their child to be obedient. (MA/030419. 100)

\subsection{What grandmother feels when raising a grandchild}

The main difficulty felt by grandmothers in caring for grandchildren is physical fatigue when they have to follow the activities of grandchildren. Subjects 1 and 2 claimed to 
be physically tired while caring for their grandchildren, in contrast to subject 2 who did not disclose this. Keep in mind that subject 2 has her job outside the home, and is not always with her grandchildren for a full day, in contrast to two other grandmothers who are with grandchildren all day. Those who accompany grandchildren all day must also adjust their activities to the activities of grandchildren. But they expressed no objection to that. They consider it is supposed to care for grandchildren like that, and caring for grandchildren is both tiring and fun. Even when grandchildren travel with their parents without a grandmother, they will want their grandchildren to go home soon.

\subsection{Childrearing agreement}

It has been discussed above that the mother-grandmother couple has the same expectations about the behavior of children, but there are variations in how they realize these expectations. Subjects 1 and 2 communicate the care of their grandchildren with their daughter. The difference is, subject 1 submits a complaint regarding the problem of grandchildren's behavior, and leaves the rest to his daughter, while subject 2 is more dominant in the application of the rules to his grandchild. Subject 3 is passive and communication between him and the child is limited to activities that must be followed by grandchildren. Subjects 1 and 3 tended to follow the behavior of their grandchildren, except that subject 1 did it out of affection and could not bear their grandchildren when they had to be hard on their grandchildren, while subject 3 did more because they did not want to bother with their grandchild's fussiness. While subject 2 more strictly limits the behavior of his grandchildren, but also tries not too hard on his grandchildren. Subjects 1 and 2 do not have clear expectations of their grandchildren and tend to leave decisions to their children and son-in-law, while subjects 2 have clearer expectations about the behavior of their grandchildren and the relationship between them, children, and grandchildren.

\subsection{Division of Labor}

The three pairs of mothers and grandmothers did not carry out specific labor division. The division of tasks occurs spontaneously, where the grandchild care activities are carried out alternately following their activities.

Well.. If I clean the house then her (granddaughter) mother will bath her. But if her mother wake up early in the morning and clean the house, then I will bath her. (NT/030419. 39-41)

\subsection{Co-parenting conflict (Support/undermining)}

All three subjects have different forms of support for their daughters in terms of care. Subject 1 tends to support what he thinks is good in terms of raising grandchildren, so there is no conflict between them. Subject 2 tends to criticize the way their children care for their grandchildren. He often said that his son was too harsh on his granddaughter, considering his grandchildren were adults, obedient and independent. Besides, he also said that his son was less responsible and less time for his grandchildren so that his grandchildren were closer to him than his daughters. However, he always positions himself as a friend to his children and son-in-law, so that there is no conflict between them. Subject 3 did not communicate much with their children related to caregiving and tended to hand over responsibilities regarding the development of grandchildren to their children.

When she cannot answer her homework, her mother will yel at her. That's why she always turn to me. I always said "Come on, don't scold her, she already independent, and she is not crybaby, why can you just be satisfy with her" (NT/030419. 89-93)

\subsection{Family Management}

In the subject family, there is no time set for doing activities together with the reason there is no time to do activities together. Even if there is vacation time, it is used for outdoor recreation activities.

We just go spontaneous. In holiday like this day, we take her to our workplace at the mall. So she will study at the mall. She is a mall girl hehehe (NT, 43-45)

\section{DISCUSSION}

From the interviews conducted, several results were concluded. First, the responsibility for care in the participant's family is held by the mother and grandmother, while the father acts as the head of the family, the decisionmaker, and the main provider of financial needs. If you look at the pattern from the perspective of gender role orientation theory. Scanzoni, states that the role structure in the family is divided into traditional and modern perspectives [30], or also called egalitarian. The division of roles is based on stratification and division of labor (division of labor) which is attached to a particular gender. In general, the division of tasks is divided based on tasks related to the internal conditions of the house (domestic tasks) and tasks related to conditions outside the home (public duties). From a traditional perspective, there is a clear stratification and division of roles between husband and wife, where the husband works in public tasks, while the wife does domestic work. In a modern or egalitarian perspective, there is a more flexible division of roles between husband and wife, where the wife also plays a role in public areas. Thus, it can be concluded that the families participating in the preliminary research mentioned above have traditional role structures. McHale et al., also mentioned that in Southeast Asian families, the conventional view of men's responsibilities in the family includes adequate income for the family and acts as a teacher, disciplinarian, and mother supporter [31]. In traditional families in Asia, it is believed that changing men's roles (such as caring for children in public) is not appropriate. Although both parents work outside the home 
to support family income, the primary responsibility for the welfare of the child rests with the mother. After the child is a child, the father does get involved in domestic activities such as traveling out of the house, eating out, putting children to sleep, or other play activities. However, the father's participation in childcare activities such as bathing, feeding, changing diapers, caring for oral health, and dressing is still very rarely done. Also, the involvement of fathers in childcare is considered temporary and only when the mother is absent.

Feinberg, stated that there were four components of coparenting, namely child-rearing agreements, division of labor, support/undermining, and family management [16]. From the results of interviews conducted, no division of labor was found in the care (division of labor) or arrangement of joint activities (family management) conducted by mothers and grandmothers in the participant's family as contained in Feinberg's co-parenting theory. Participants revealed that caregiving is done without a clear division of tasks and follows more conditions, for example, if the mother is at home then the mother feeds the child while if the mother is not at home then the grandmother feeds the child. Besides, they do not organize special activities where all family members can interact with each other because some parents work with the shift system, so it is not certain the time available to gather together. This shows that co-parenting theory in families with characteristics such as the participant's family need to be reviewed.

The results of interviews that show that co-parenting in the subject's family is carried out by mothers and grandmothers is in line with the results of Pujiatni \& Kirana's research that shows the characteristics of multigenerational families in Indonesia that are different from others in terms of involvement in childcare [32] [33]. Grandparents (more often grandmothers) are described as more active and directly involved in the care of their grandchildren [34]. The same characteristics with Indonesian families are also found in families in Asian countries such as China [35], Japan and Singapore [36].

When viewed from the aspect of support and conflict, in carrying out co-parenting there are couples of mothers and grandmothers who can work well together, where differences in the care they carry out are discussed together, each accepting each other's opinions, so as not to cause conflict. However, in other families, there is a conflict between mothers and grandmothers about good parenting techniques for children and the lack of involvement of mothers in childcare. However, the conflict does not lead to open conflict (for example arguing in front of children or making sarcastic remarks about their parenting behavior). The non-emergence of open conflict is related to the fundamental values in the family structure in Asia that place parents as parents' parties who are held and respected unconditionally [37]. Also, in the philosophy of Javanese family life, conflict is something that should be avoided, because the main value of life in harmony and mutual respect [38]. However, the existence of the conflict and its effects seems inevitable.

In conflicting families, grandmother stated that mothers do not have many roles in caring for children, pay less attention to children, and are too harsh on children. From the mother's side, she stated that the grandmother lacked trust in the mother, and considered the mother to be incompetent in conducting care, even though in reality she revealed that she had tried to carry out care properly. In this conflicting family, it is also found a pattern of parenting that tends not to warm up to the child, and treats the child harshly, such as shouting or punishing if a child makes a mistake. Meanwhile, the mother claimed to do this for fear that the child would behave disrespectfully if the mother was too soft on them.

\section{CONCLUSION}

This study illustrates how the experience of mothers and grandmothers in working together to care for children. Further research can be carried out to deepen how these behavioral patterns can be formed, especially in times of changing parental status as an important phase in parenting practice. Explanation of the dynamics of existing coparenting relationships is still limited to the dynamics of coparenting between father and mother in the nuclear family. While multigenerational families are a fairly common form of family in Indonesia, so the study of co-parenting in multigenerational families in Indonesia still needs to be done.

\section{ACKNOWLEDGMENT}

The author received financial support from Universitas Muhammadiyah Surabaya for publication of this article.

\section{REFERENCES}

[1] U. Bronfenbrenner, "Toward an experimental ecology of human development," American psychologist, vol. 32, no. 7, p. 513, 1977.

[2] U. Bronfenbrenner, "Ecology of the family as a context for human development: Research perspectives," Developmental psychology, vol. 22, no. 6, p. 723, 1986.

[3] U. Bronfenbrenner, Making human beings human: Bioecological perspectives on human development., California : Sage Publication, Inc., 2005.

[4] P. Minuchin, "Families and individual development: Provocations from the field of family therapy," Child development, pp. 289-302, 1985.

[5] C. H. Mindel, "Multigenerational family households: Recent trends and implications for the future," The Gerontologist, pp. 456-463, 1979.

[6] V. L. Bengtson, "Beyond the Nuclear Family: The Increasing Importance of Multigenerational Bonds: The 
parents with mentally retarded and typically developing children," Child Development, vol. 62, p. 1434-1448., 1991.

[19] R. S. \&. W. S. H. Cohen, "The parenting alliance," in Parenthood: A psychodynamic perspective, New York, Guilford, 1984, p. 33-49.

[20] L. A. \&. H. D. P. Van Egeren, "Coming to terms with coparenting: Implications of definition and measurement," Journal of Adult Development, vol. 11, no. 3, pp. 165-178, 2004.

[21] J. M. J. S. A. \&. C. D. Baker, "Mothergrandmother coparenting relationships in families with incarcerated mothers: a pilot investigation," Family process, vol. 49, no. 2, pp. 165-184, 2010.

[22] M. A. M. W. R. G. H. C. M. \&. F. L. P. K. I. Barnett, "Mother-Grandmother Conflict, Negative Parenting, and Young Children's Social Development in Multigenerational Families.," Family Relations, vol. 61, no. 5, 2012.

[23] X. \&. L. Y. Li, "Parent-Grandparent Coparenting Relationship, Maternal Parenting Self-efficacy, and Young Children's Social Competence in Chinese Urban Families," Journal of Child and Family Studies, pp. 1-9, 2019.

[24] M. S. K. A. L. N. C. S. S. J. \&. G. L. D. Spencer, "Multigenerational coresidence and childrearing conflict: Links to parenting stress in teenage mothers across the first two years postpartum," Applied Developmental Science, 2002.

[25] M. H. Bornstein, "Cultural approaches to parenting," Parenting, vol. 12, no. 2-3, pp. 212-221, 2012 .

[26] J. P. McHale, "Overt and covert coparenting processes in the family," Family process, vol. 36, no. 2, pp. 183-201, 1997.

[27] R. H. \&. C. R. F. Bradley, "Socioeconomic status and child development," Annual review of psychology, vol. 53, no. 1, pp. 371-399, 2002.

[28] V. C. McLoyd, "Socioeconomic disadvantage and child development," American psychologist, vol. 53, no. 2 , p. $185,1998$.

[29] M. \&. H. A. M. Miles, Qualitative Data Analysis, 2nd edition, London: Sage Publication, 1994.

[30] J. Scanzoni, "Contemporary marriage types: A research note," Journal of Family Issues, vol. 1, no. 1, pp. 125-140, 1980.
[18] F. J. \&. Z. D. E. Floyd, "Marriage and the parenting partnership: Perceptions and interactions of

[17] F. M. Deutsch, "Equally shared parenting," Current Directions in Psychological Science, vol. 10, p. 25-28, 2001.

[16] M. E. Feinberg, "The internal structure and ecological context of coparenting: A framework for research and intervention," Parenting: Science and Practice, vol. 3, no. 2, pp. 95-131., 2003. 
[39] M. E. Feinberg, "Coparenting and the transition to parenthood: A framework for prevention," Clinical child and family psychology review, vol. 5, no. 3, pp. 173-195, 2002.

[40] J. P. McHale, "Coparenting and triadic interactions during infancy: The roles of marital distress and child gender," Developmental psychology, vol. 31, no. 6, p. 985, 1995.

[41] J. P. \&. L. K. M. McHale, "Coparenting: A conceptual and clinical examination of family sistems.," American Psychological Association., 2011.

[42] J. P. K.-H. R. L. A. \&. R. J. L. McHale, "Parental reports of coparenting and observed coparenting behavior during the toddler period," Journal of Family Psychology, vol. 14, no. 2, p. 220, 2000.

[43] S. J. M. S. C. F. C. A. \&. M. J. L. SchoppeSullivan, "Associations between coparenting and marital behavior from infancy to the preschool years," Journal of Family Psychology, vol. 18, no. 1, p. 194, 2004.

[44] J. P. \&. R. T. McHale, "Is seeing believing?: Expectant parents' outlooks on coparenting and later coparenting solidarity," Infant Behavior and Development, vol. 30, no. 1, pp. 63-81, 2007.

[45] D. M. J. T. J. P. M. \&. K. R. Elliston, "Withdrawal from coparenting interactions during early infancy," Family process, vol. 47, no. 4, pp. 481-499, 2008.

[46] D. J. J. A. M. S. C. R.-G. W. B. G. L. \&. S.-S. S. J. Laxman, "Stability and antecedents of coparenting quality: The role of parent personality and child temperament.," Infant Behavior and Development, 2013
[38] F. Magnis-Suseno, Etika Jawa : Sebuah Analisa Falsafi tentang Kebijaksanaan Hidup Jawa, Jakarta :

Gramedia, 1984.
37] Y. W. C. M. \&. L. P. A. Ying, "Family adolescents," Cultural Diversity and Ethnic Minority Psychology, vol. 5, no. 4, p. 350, 1999. 